\title{
Feature
}

\section{Adaptive Multi-Standard Circuits and Systems for Wireless Communications}

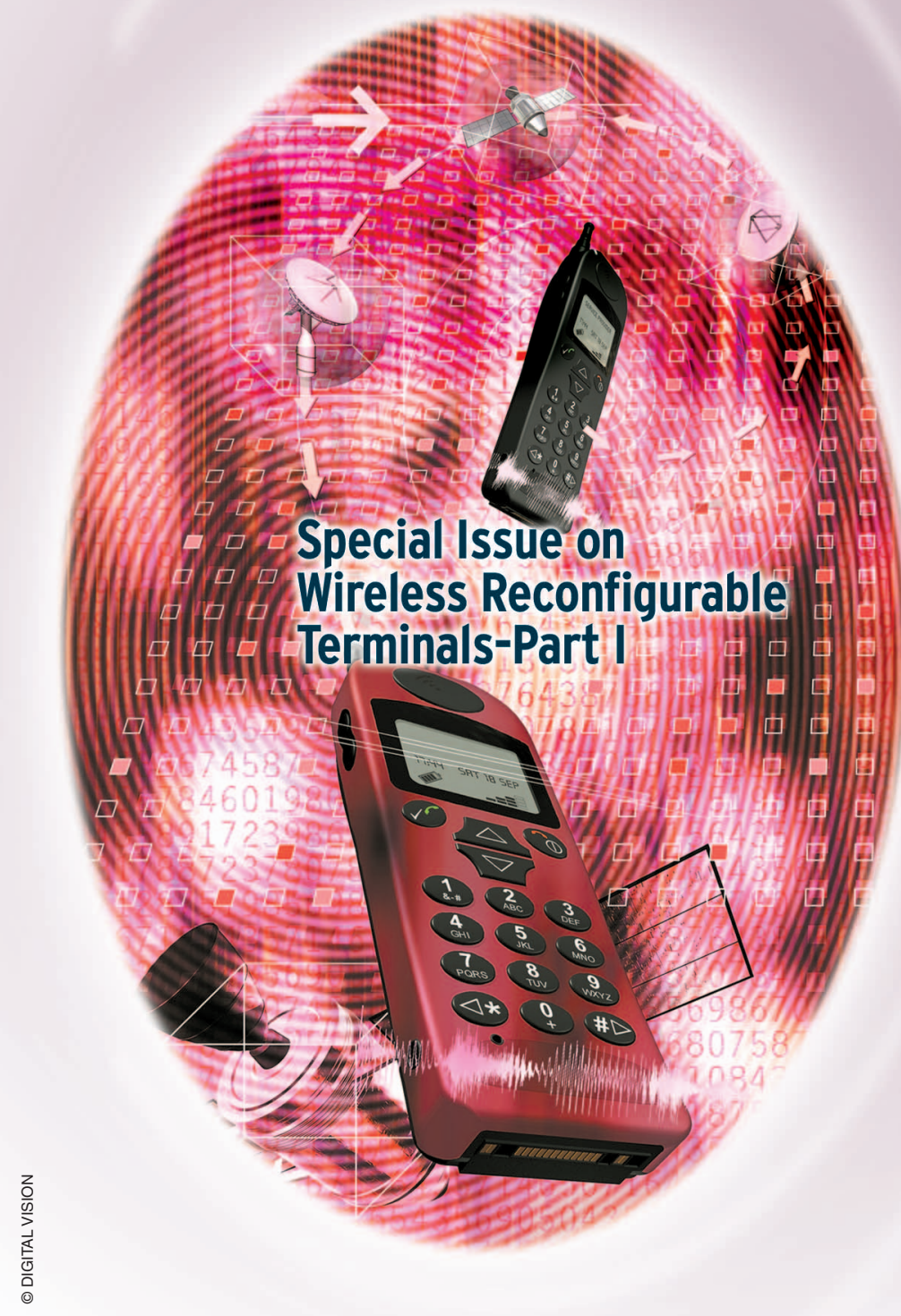

Aleksandar Tasić, Wouter A. Serdijn, and John R. Long

\section{Abstract}

Telecom trends such as smooth migration towards higher data rates and higher capacities for multimedia applications, and provision of various services (text, audio, video) from different standards with the same wireless device require integrated designs that work across multiple standards, can easily be reused and achieve maximum hardware share at minimum power consumption. This can be achieved by using adaptive circuits that are able to trade off power consumption for performance on the fly. Realization of the adaptivity function requires scaling of parameters such as current consumption to the demands of the signalprocessing task. This paper describes adaptivity design concepts and application of design for adaptivity to multi-standard circuits and systems for wireless communications. An exploratory multi-standard RF front-end is discussed with phase-noise tuning, noise-figure tuning, and input-intercept point tuning requirements of $21 \mathrm{~dB}$, $12 \mathrm{~dB}$, and $7 \mathrm{~dB}$, respectively. 


\section{Introduction}

$\mathrm{T}$ he demands for new telecom services requiring higher capacities and data rates have motivated the development of new-generation broadband wireless systems. The coexistence of different cellular systems requires multi-mode, multi-band, and multi-standard mobile terminals. To prolong talk-time, it is desirable to share transceiver building blocks in these multifunctional handsets.

Transceivers for multi-mode and multi-standard telephony are most often implemented by replicating the receiver circuits for each operating band or standard. This allows applications such as GSM and WCDMA to operate concurrently (i.e., one can receive or make a call with either system at any time). Although high level of integration is possible on silicon, the increase in RF hardware required to implement this type of multi-standard radio increases the total current consumption, thereby reducing talk time. Transceivers that in such situations can share circuit functions between different standards in an adaptive manner have the advantages of low power consumption, small chip area, long talk time, and, most importantly, have the potential for low cost [1].

The following section advocates for the application of adaptivity to multi-standard low-power wireless RF circuits. $\mathrm{RF}$ amplifiers' and oscillators' adaptivity phenomena are introduced in Section III. The application of design for adaptivity to multi-standard oscillators and RF front-ends is discussed in Section IV. Section V draws the conclusions.

\section{Adaptive Multi-Standard Low-Power Wireless RF Circuit Design}

Progress in silicon IC technology [2] and innovations in IC design have enabled mobility of wireless consumer products and services. Having started out with limited

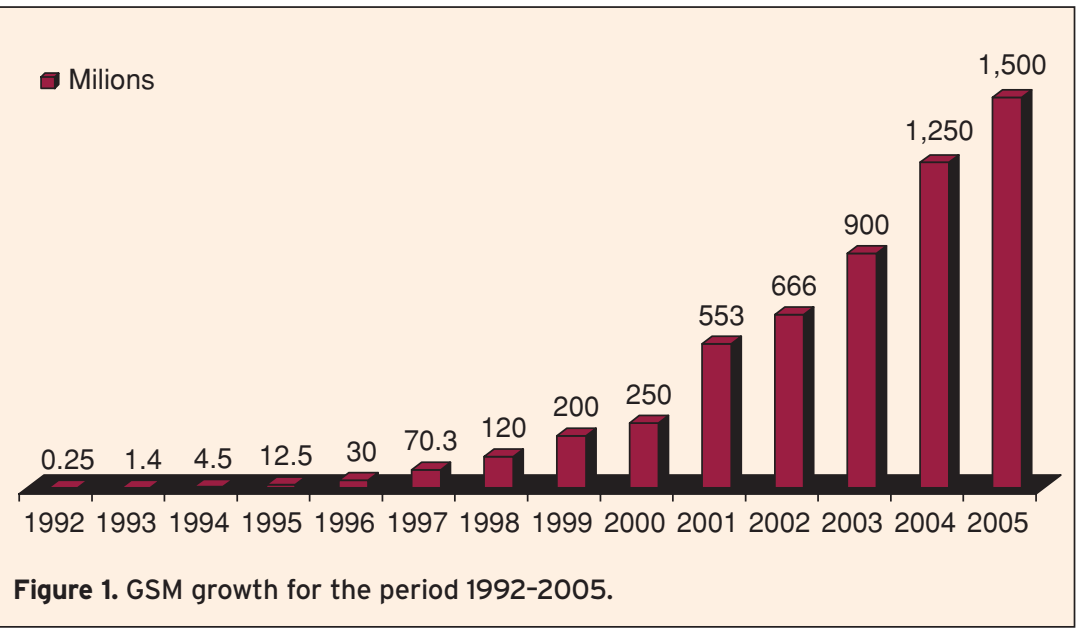

performance capabilities beyond simple telephony, mobile communications technologies are now entering all aspects of our lives.

An example of the enormous expansion of the wireless market is shown in Figure 1. The total number of global mobile users amounts to 2 billion (third quarter 2005), whereas the number of GSM (Global System for Mobile communications) users is estimated at 1.5 billion [3].

Despite the expansive sales growth of wireless devices, the use of wireless services generates even greater profit for telecom companies. Furthermore, even larger growth requires many new services provided by mobile equipment (e.g., MMS, web access, and e-mail).

Aside from the mobile phone market [2,3], there are many other wireless applications. Wireless connections to wired computer networks have become feasible. Wireless systems allow for cost-effective installation and deployment of electronics equipment by obviating the need for wires and cables. Wireless RF systems will undoubtedly spawn telemedicine, that is, remote, wireless medical monitoring. An intelligent transportation system that allows for communication and traffic control on the highway is yet another example of a mass market for wireless technology in the future. However, to support all these applications, more sophisticated RF devices are required.

\section{A. Low-Power and Adaptive RF Circuit Design}

The communication devices of today and the future will not only have to support applications ranging from text, telephony, audio, and graphics to video, but they will also have to maintain connections with many other devices in a variety of environments (and not only with a single base station). Moreover, they should be position aware, and perhaps wearable than just portable [4].

A combination of multiple functional requirements and a small energy supply is an argument for the design of both adaptive low-power (i.e., poweraware) hardware and adaptive lowpower software. Simply stated, as consumer demands outstrip the cost benefits achieved by Moore's Law and low-power circuit design, a new design direction is found in adaptivity. This eventually leads to smaller physical size, longer standby and active times, and enhanced functionality of mobile wearable devices.

The quality of service of mobile devices changes with the position and

Aleksandar Tasić, Wouter A. Serdijn, and John R. Long are with the Electronics Research Laboratory, Delft University of Technology, The Netherlands, Phone: +31 (0)15 278 1579; Fax: +31 (0)15 278 5922; E-mail: a.tasic@ewi.tudelft.nl 
speed of mobile users. It also depends on the application, the number of users in a cell as well as their activity. A mobile device must handle the variable context efficiently due to scarce resources, especially limited battery power.

A power-aware (i.e., adaptive) RF design approach poses unique challenges: from hardware design to application software, throughout all layers of the underlying communication protocol (i.e., the processing technology, device level, circuit level, system level, as well as protocol level, software and application levels).

A block diagram of the receive part of an adaptive mobile device is shown in Figure 2. This receiver consists of an analogue RF front-end, analogue baseband electronics (analogue processing of the received signal), and a digital back-end consisting of a dedicated central processing unit (CPU) and a memory.

Whereas the transceiver circuits determine instantaneous power consumption, the average consumption depends on the power management of the complete system $[5,6]$. This implies that not only local, but also global (in all layers and at all time) power optimization and awareness are important for extending the "lifetime" of mobile devices (i.e., the time between battery recharges).

$\mathrm{RF}$ and power management have become the fastest growing segments in wireless IC revenue, due to the integration and increasingly complex power requirements, which are driven by advancing functionality (e.g., video, text) and transmission speeds in wireless devices. The RF portion is estimated at $19 \%$ of the wireless IC market [7].

Setting the performance parameters of an RF frontend by means of adaptive RF front-end circuitry [1] is a way to manage power consumption in the RF path of a receiver. Adaptive RF front-end circuits (shown in Figure 2), viz., an adaptive low-noise amplifier, an adaptive voltage-controlled oscillator and an adaptive mixer, allow efficient use of scarce battery resources, thereby extending the lifetime of a mobile device. Furthermore, power-conscious adaptive analogue baseband and digital back-end circuits enable complete hardware adaptivity.

$\mathrm{RF}$ front-end robustness can be further improved by control of symbol rates, antenna beam patterns, transmitter power levels, and by control of circuit noise and linearity levels. For example, adaptive modulation and adaptive coding strategies [8], where the system can choose an optimal modulation and coding technique based on the temporal circumstances, can ameliorate the effects of multi-path fading, shadow fading, and path loss.
Graphical interaction with our direct environment combined with mobility is another intriguing concept in which power-conscious RF circuit design plays an important role [9]. If a lightweight video camera is attached to a mobile display for position tracking and recording of video, the hardware complexity must be reduced in order to keep the power consumption low. Since the RF front-end cannot operate with scarce resources, the power consumption can be reduced by limiting the processing and memory capabilities of the headset unit. In turn, this requires "clever" (power-aware) processing of received and transmitted data.

At an even higher hierarchical level, an example of a power-aware software implementation is the efficiency of a compiled code [10]. An example of application-level adaptivity is scaling the operating power and clock frequency in a general-purpose CPU under the control of power-aware applications, such as video- and audiodecoding software. Here, dynamic adjustment of the supply voltage can be traded for processor speed, allowing considerable power savings in the digital circuitry [11].

A framework for the exchange of performance and power consumption information between $\mathrm{RF}$ receiver, hard disk, CPU, operating system and the application has been developed within the Ubiquitous Communications project [6]. It is an example of a fully adaptive low-power mobile system.

\section{B. Multi-Standard and Adaptive RF Circuit Design}

Continuous migration towards higher data rates and higher channel capacities and provision of various services (such as text, audio and video) for multimedia applications require not only adaptive and low power designs, but also the designs that work across multiple bands and standards.

Multi-standard receivers typically use duplicate circuit blocks, or even entire radio receivers for each standard. Although this approach is simpler to implement, it is neither optimal in cost nor in power consumption [12]. When different standards do not operate simultaneously, circuit blocks of a multi-standard handset can be shared. By

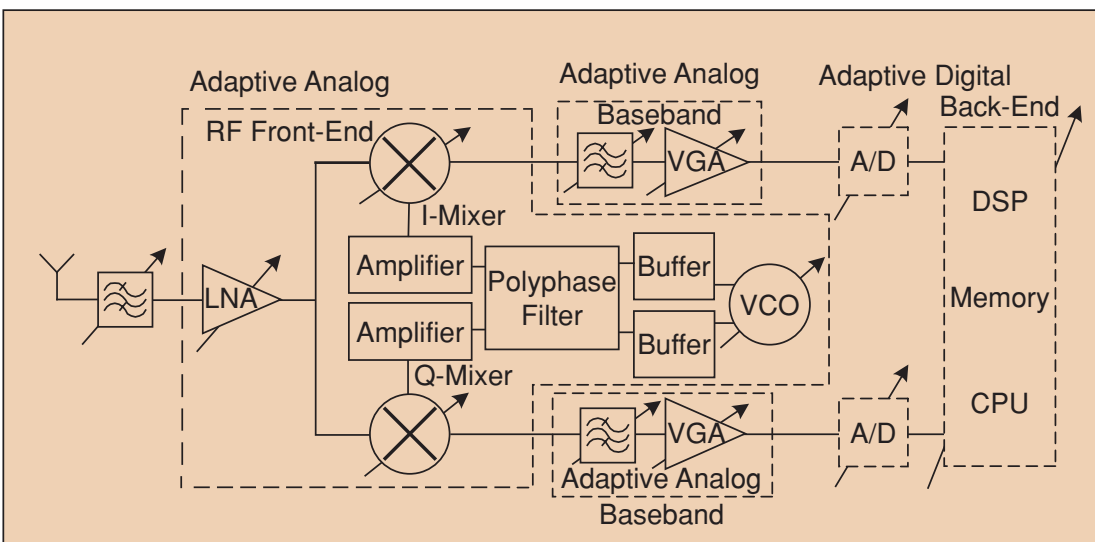

Figure 2. Block diagram of an adaptive receiver. 
using circuits that are able to trade off power consumption for performance on the fly, i.e., adaptive multi-standard circuits, considerable power can be saved. There is currently an apparent migration in RF IC design towards multi-mode multi-band integrated modules for low-noise amplifiers [13], oscillators [14], power amplifiers [2] and transceivers [1]. Design of multi-standard oscillators and multi-standard front-ends is discussed in this paper.

In addition to multimode capability at radio frequencies, adaptivity should be implemented at baseband frequencies as well. After a signal is downconverted to the baseband, it must be filtered, amplified and digitized. In order to accommodate multiple radio standards with different bandwidths and modulation schemes, such receivers require different channel and image-reject filter bandwidths and different analogue-to-digital converter (ADC) resolutions. For example, a variable-bandwidth baseband filter and variable-resolution ADC can be used to alternate between different modes of operation [15].

Finally, because adaptive multi-standard low-power RF front-ends are able to share building blocks across different standards, they have advantages over their predecessors: they use a smaller chip area, and most importantly, have a potential for lower overall cost.

\section{Adaptivity of RF Circuits}

The variant nature of radio-channel conditions and variable requirements imposed on RF circuits in the direct signal path urge for designs that can respond to changes "on-the-fly" (i.e., adaptive designs). Adaptivity phenomena and design concepts of low-noise amplifiers and voltagecontrolled oscillators are described in this section. They establish relationships between performance parameters of both amplifiers and oscillators, respectively.

\section{A. Adaptivity of Low-Noise Amplifiers}

Adaptivity figures of merit (AFOM) (i.e., figures characterizing circuits' adaptivity phenomena) [1] of low-noise amplifiers (LNA) describe the relationships and trade-offs between the performance parameters, being noise figure, gain, linearity and power consumption. They form the basis for the design of LNAs that operate across multiple standards.

Some of the low-noise amplifier adaptivity phenomena are: noise-figure, linearity, and gain tuning. Corresponding adaptivity figures of merit are: gain tuning range (GTR), noise-figure tuning range (NFTR), and tuning range of input-referred 3rd-order intercept point (IIP3TR). Adaptivity phenomena and their figures of merit describe the change of circuit performance parameters with respect to power consumption.

In order to determine the range of amplifier's adaptivity, the maximum and the minimum power consumption levels have to be determined.
Accordingly, the minimum biasing point, which determines the minimum power consumption, depends on both LNA and RF front-end system specifications: it is the power level that provides acceptable dynamic range and sensitivity of a complete system, with satisfactory noise figure, gain and linearity of an amplifier. This mode of operation can be chosen when environmental (channel) conditions improve, i.e., a receive desired signal is stronger and interference signals are weaker. In a multistandard system, an amplifier operates in this state when a standard with relaxed requirements is active.

On the other hand, the maximum power consumption level depends on the RF front-end worst-case condition specifications as well as the system power budget: how much power can be "burned" in the amplifier and RF system. This mode of operation can be chosen when, for example, a receive signal is rather weak. The amplifier performance requirements in this situation are large gain and good noise figure and linearity. This would also correspond to the operation in a most-demanding mode of a multi-standard system.

For example, demanding linearity and noise-figure (and power consumption) requirements are due for the highperformance WCDMA standard [16], whereas for the lowperformance DECT standard [17], the power consumption, noise and linearity requirements are rather relaxed.

The NFTR, GTR and IIP3TR relate to the $N F$, gain and IIP3 differences between the corresponding maximum (i.e., demanding mode of operation) and minimum (i.e., relaxed mode of operation) power consumption levels.

\section{B. Adaptivity of Voltage-Controlled Oscillators}

Oscillators' adaptivity phenomena encompass phase-noise tuning and frequency-transconductance tuning. They can be qualitatively and quantitatively described by means of their figures of merit. Phase-noise tuning range describes phase-noise adaptivity of an oscillator with respect to power consumption. Frequency-transconductance sensitivity describes the effect of compensating for the change in the oscillation condition due to frequency tuning.

\section{B.1. Phase-Noise Tuning}

If the radio-channel conditions improve (or a relaxed communication standard is active), poorer phase noise of oscillators may be tolerable, allowing for power savings. Designing for adaptivity [18] is in such a situation a better solution than a "fixed" design practice, which is "blind" and "deaf" for the volatile specifications imposed by the air interface.

By trading phase noise for power consumption, oscillators and oscillating systems can be adapted to varying conditions and thereby satisfy the requirements of the complete RF front-end system. The concept of phase-noise tuning [18] shows explicitly how phase noise and power consumption trade between each other in an adaptive way. 
An adaptive VCO [14] is shown in Figure 3. It consists of a resonant $\mathrm{LC}$ tank ( $L$ is the tank inductance, $C_{V}$ the tank varactor capacitance) and a cross-coupled transconductance amplifier $\left(Q_{1}, Q_{2}\right)$. The bias tail-current source provides current $I_{\text {TAIL }}$.

In adaptive oscillators the small-signal loop gain $(k)$, voltage swing and phase noise can be varied by changing current $I_{\mathrm{TAIL}}$. This allows adaptation of the oscillator's phase noise to different conditions. Parameter $k$ defines how far the oscillator is from the start-up condition being $k=1$. In addition, $k$ relates to the excess of the negative conductance necessary for the compensation of the losses in the LC-tank. Namely, if the tank conductance is $G_{T K}$, then for the start-up of the oscillations, the equivalent negative conductance seen by the LC-tank must be $G_{M, T K}=k G_{T K}$, with $k$ larger than one. In turn, a $k$-times larger negative conductance of the active part of the oscillator requires a $k$-times increase in power consumption with respect to the start-up condition. Therefore, we relate the change in the small-signal loop gain $k$ to the change in power consumption.

The figure of merit describing the oscillator's adaptivity to phase noise (phase-noise tuning range, PNTR) is derived in [14] for a $k_{2} / k_{1}$-times change in power consumption. For example, for a factor $12\left(k_{2} / k_{1}\right)$ change in power consumption, a phase-noise tuning range of $20 \mathrm{~dB}$ can be achieved [14].

\section{B.2. Frequency-Transconductance Tuning}

For low-power voltage-controlled oscillators, a design is usually aimed at a loop gain slightly larger than the necessary minimum of one (e.g., two). In such cases, an increase in the capacitance of the oscillator's LC-tank varactor in order to lower the oscillation frequency results in an increase of the effective tank conductance. If the design is "fixed" rather than adaptive, the oscillation condition deteriorates as the loop gain is lowered. Accordingly, this can bring an RF frontend to a halt, as there might be no oscillations.

In situations where power consumption is of less concern than oscillator phase noise, the repercussions are different but not less detrimental. The oscillation condition is rather relaxed, as the loop gain can be much larger than two. However, the voltage swing over the LC-tank will be reduced due to the increase in the effective tank conductance (increased varactor capacitance), resulting in potentially poorer phase-noise performance.

In both of these examples, the oscillator could still fulfill the requirements if the bias conditions of the transconductor transistors were adapted (i.e., modified in a controlled fashion). Thus, frequency-transconductance ( $\left.C-g_{m}\right)$ tuning [18] is the control mechanism compensating for the change in the VCO LC-tank characteristic (whose conductance is changed due to frequency tuning) by varying the oscillator's bias conditions (i.e., the transconductance $g_{m}$ ).
The concept of $C-g_{m}$ tuning illustrates the relationship between the varactor diode tuning voltage $U_{T}$ and the biasing tail current $I_{\mathrm{TAIL}}$, both indicated in Figure 3 . The resulting sensitivity of the tail current to the tuning voltage shows to what extent the biasing condition should be changed in response to a change in the frequency, in order to keep the oscillator operating under the specified conditions.

For example, a sensitivity of $0.25 \mathrm{~mA} / \mathrm{V}$ infers that in order to sustain the oscillations under the same condition (i.e., the same loop gain) the tail current should be either increased or reduced (depending on the direction of the frequency tuning) by $0.25 \mathrm{~mA}$ for a $1 \mathrm{~V}$ change in a varactor voltage. The counterpart of the $C-g_{m}$ tuning in the circuitry is an amplitude control mechanism, as constant loop gain (operation condition) means constant amplitude of the signal across the LC-tank of the oscillator.

\section{Application of Design for Adaptivity to Multi-Standard Voltage-Controlled Oscillators and RF Front-Ends}

Today's portable communication devices enable a growing variety of applications, ranging from text messaging, telephony and MP3 audio to full video. These devices must maintain connectivity while running multiple applications, they must track position, and (in the near future) be wearable rather than just portable. However, the energy supply for portables is fixed by the size and weight of the batteries in a hand-held device. Consequently, the current consumption of circuitry in multistandard and multi-functional hand-helds must be reduced in order to meet these increasing functional and concurrent operational requirements.

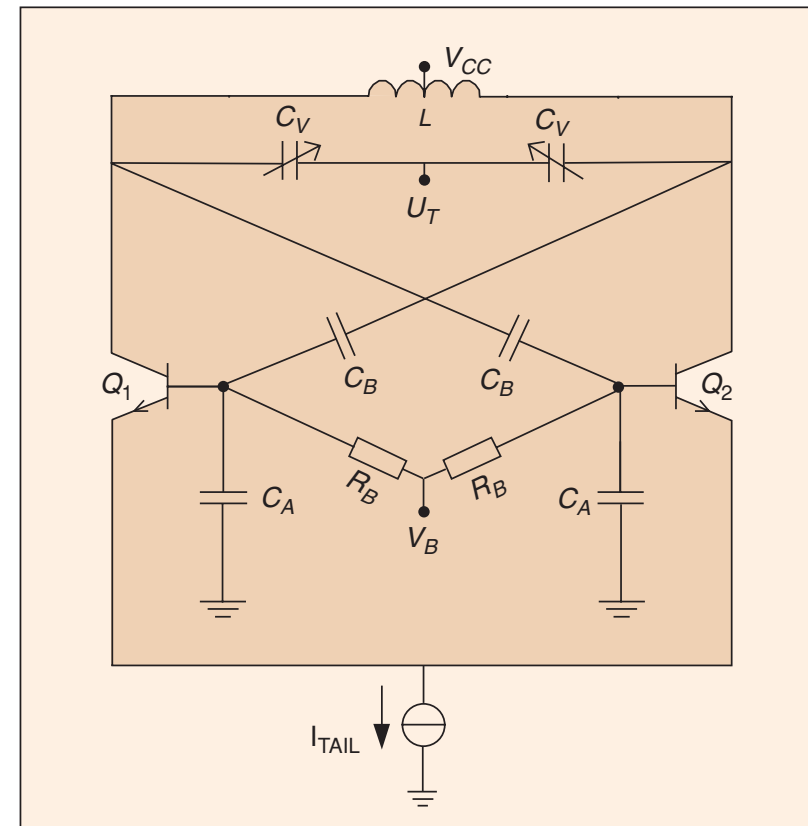

Figure 3. An adaptive LC-oscillator. 
Table 1.

Multi-standard VCO requirements.

\begin{tabular}{lccccc} 
MSA-VCO & DCS1800 & WCDMA & WLAN & Bluetooth & DECT \\
\hline PN@1 MHz [dBc/Hz] & -123 & -110 & -110 & -110 & -100
\end{tabular}

outlined in this section. A factor of 12 reduction in power consumption is realized, with a phase-noise tuning range of $20 \mathrm{~dB}$ by adapting the VCO bias to the desired application. The $\mathrm{VCO}$ achieves $-123 \mathrm{dBc} / \mathrm{Hz}$ and $-103 \mathrm{dBc} / \mathrm{Hz}$ phase noise at $1 \mathrm{MHz}$ offset in

Multi-standard modules (MSMs) can be implemented in different ways:

- MSMs can be implemented as standalone fixed circuits that are designed for the worst-case condition of the most demanding standard [19]. Even though operating conditions might improve or a less demanding standard might be active, they always operate at the highest power consumption levels. This design approach is therefore power inefficient.

- MSMs can be implemented as multiple circuits, i.e., one per standard [12]. Even though simple to implement, this approach requires more silicon area, and is therefore area inefficient. Moreover, when multiple standards operate simultaneously, power consumption increases.

- MSMs can be implemented as standalone, adaptive circuits, by sharing circuit functions across multiple standards [14] when they do not have to operate simultaneously. This allows for reduced area and power consumption and, most importantly, has the potential for reduced cost. Circuit adaptivity requires scaling parameters such as current consumption to the demands of the signal-processing task at hand.

\section{A. Multi-Standard Adaptive Voltage-Controlled Oscillators}

An adaptive, multi-standard/multi-band voltage-controlled oscillator that satisfies phase-noise requirements in receive mode of some 2 nd and 3rd generation wireless standards (DCS1800/WCDMA/WLAN-Bluetooth-DECT) is

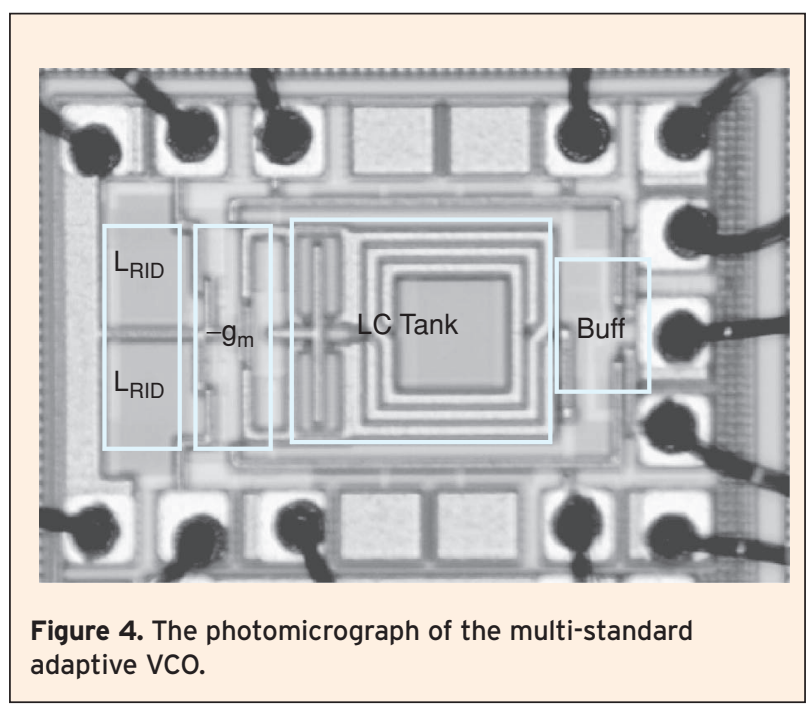

a $2.1 \mathrm{GHz}$ band at supply currents of $6 \mathrm{~mA}$ and $0.5 \mathrm{~mA}$, respectively [14].

Selection of parameters for the adaptive multistandard oscillator and experimental results are discussed next.

\section{A.1. Selection of Parameters for MSA-VCO}

The receiver phase-noise requirements (expressed in $\mathrm{dBc} / \mathrm{Hz}$ at $1 \mathrm{MHz}$ offset) for five different standards (i.e., DCS1800/WCDMA/WLAN-Bluetooth-DECT) are listed in Table 1 [16, 17, 20-22]. We will refer to the DCS1800 standard as a phase-noise demanding (PN-D) standard, to the WCDMA, WLAN and Bluetooth standards as phase-noise moderate (PN-M) standards, and to the DECT standard as a phase-noise relaxed (PN-R) standard.

The VCO shown in Figure 3 is used to implement the multi-standard adaptive oscillator. Given the phase-noise requirements listed in Table 1 , the phase-noise range between the demanding (PN-D) and relaxed (PN-R) modes is $P N T R=21 \mathrm{~dB}$. From the phase-noise and frequency tuning requirements, the LC-tank parameters can be determined, i.e., coil inductance and varactor capacitance. Once the resonator parameters are known, the tank conductance and power consumption (i.e., tail current) can be calculated. The choice of the base-bias voltage $V_{B}$ is a compromise between a large output voltage swing and saturation of transconductor devices $Q_{1}$ and $Q_{2}$.

The chip photomicrograph of the multi-standard VCO is shown in Figure 4. It occupies an area of $400 \times 750 \mathrm{um}^{2}$, without the output buffer and bondpads. Wire-bonded in a 20 lead RF package, the chip is tested in a metal fixture with filtering on all bias and supply lines [14].

For a $3 \mathrm{~V}$ supply, a frequency tuning range of $600 \mathrm{MHz}$ (i.e., output from $1.8 \mathrm{GHz}$ to $2.4 \mathrm{GHz}$ ) is measured. Plots of the measured phase noise at $1 \mathrm{MHz}$ offset in the 2.1 $\mathrm{GHz}$ mid-frequency band are shown in Figure 5 for two operating conditions.

As can be seen from Figure 5, by adapting the bias tail current between $0.5 \mathrm{~mA}$ and $6 \mathrm{~mA}$, a phase-noise tuning range of $20 \mathrm{~dB}$ has been achieved.

\section{B. Multi-Standard Adaptive RF Front-Ends}

In this section, the results of an exploratory system analysis for a multi-standard adaptive (MSA) image-reject (IR) quadrature downconverter (QD) are discussed. The MSA-IR downconverter (oscillator and mixers) satisfies the requirements of the considered 2nd and 3rd generation 
standards. This design allows for adaptation between different standards by trading RF performance for current consumption [23].

Concurrent operation of different wireless standards using a common $\mathrm{RF}$ receiver poses demands on performance (e.g., band selection, image-rejection and noise/ power match prior to low-noise amplification) that are difficult to meet using a single RF path [13]. Therefore, multistandard receivers often use duplicate circuit blocks, or even multiple RF front-ends (i.e., one for each standard).

The multi-standard front-end, shown in Figure 6, is a compromise between these two approaches. The MSA-IR downconverter test circuit consists of an adaptive voltage-controlled oscillator, oscillator buffers, a two-stage poly-phase filter to generate in-phase (I) and quadraturephase (Q) local oscillator signals, mixer buffer amplifiers and dual balanced mixers.

Impedance matching, packaging and prefiltering requirements are relaxed and simplified by using multiple low-noise amplifiers. A single quadrature or image-reject downconverter can then be used to interface the RF and baseband sections of the receiver. An RF switch is used to select the standard of interest. If the $\mathrm{VCO}$ and mixer performance are adequate to cover the range of signals anticipated for each application, the downconverter enables a multistandard front-end realization with a single circuit block (the MSA-QD IC in Figure 6).

Analog and digital baseband signal processing functions could be used to monitor quality of service (e.g., error rate of the detected bit sequences) and adjust the receiver parameters (e.g., tune a single bias current or multiple currents) in real-time to meet the requirements of a given standard.

The following sections discuss the requirements and selection of performance parameters for multi-standard adaptive RF frontend circuits.

\section{B.1. Performance Requirements for MSA RF Front-Ends}

The noise (i.e., noise-figure and phase-noise) and linearity require- ments (i.e., IIP3) for $1.8 \mathrm{GHz}-\mathrm{DCS} 1800,2.1 \mathrm{GHz}-\mathrm{WCDMA}$, and $2.4 \mathrm{GHz}$ WLAN(802.11b)/ Bluetooth/DECT standards are listed in Table 2 [15-17, 20-22, 24-26]. The MSA front-end operating modes are classified with respect to phase noise, noise figure and linearity requirements as: demanding (D) for DCS1800 and WCDMA, moderate (M) for 802.11, and relaxed (R) for Bluetooth and DECT standards. The noise and linearity requirements are referred to the input of an LNA. These standards are chosen to show the feasibility of the adaptivity concepts for multistandard applications.

Design procedure for an adaptive multi-standard circuit is different from the design for a single standard. Figures of merit referring to a number of operating conditions and specifications are required for multi-standard designs, i.e., the adaptivity figures of merit. For oscillators, the phase-noise tuning range is used to specify the difference between the maximum and minimum
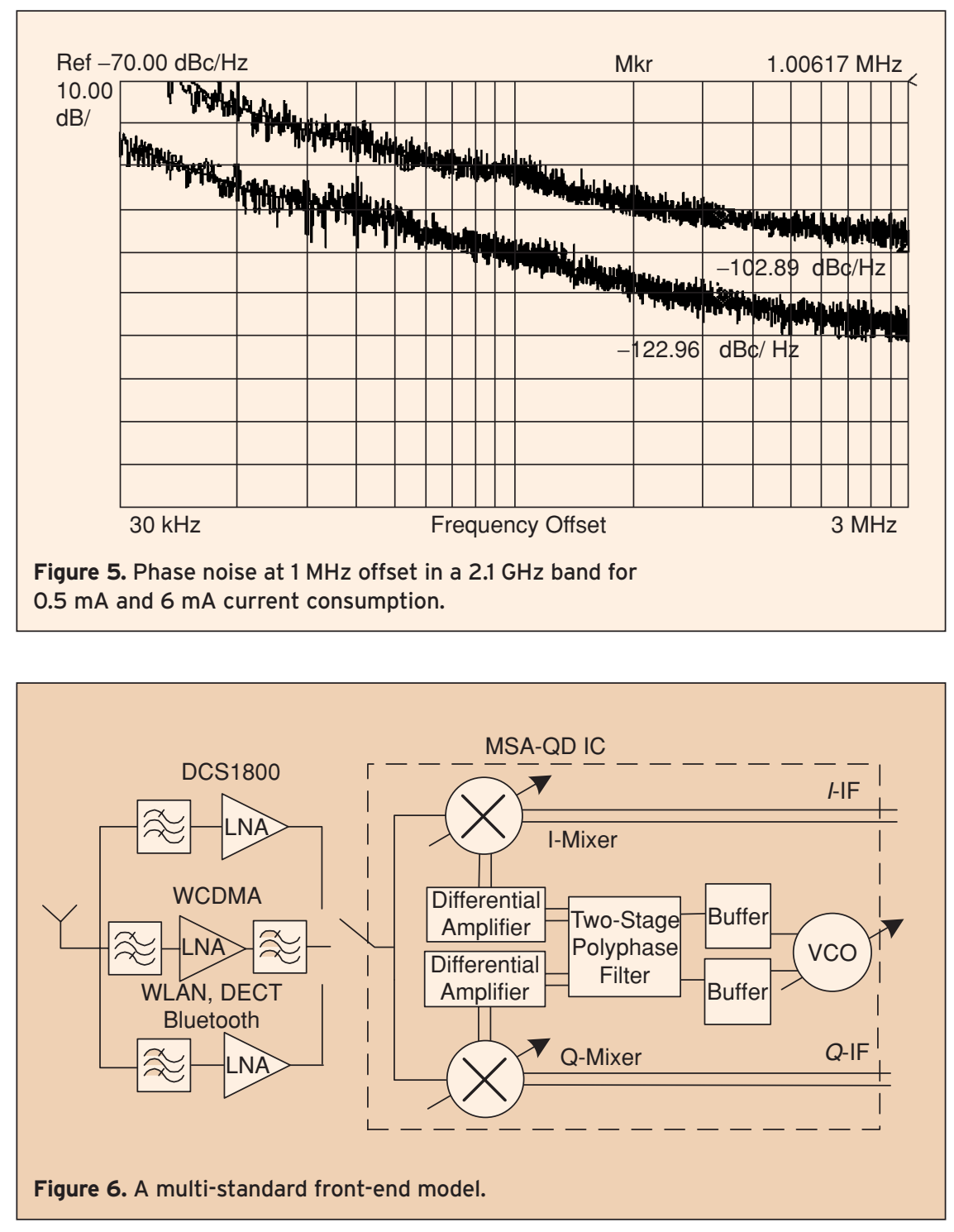
Table 2.

Performance requirements for different modes (desired specifications).

\begin{tabular}{|lccc|}
\hline Specification/Mode & Demanding & Moderate & Relaxed \\
\hline$N F_{D}[\mathrm{~dB}]$ & 6 & 10 & 18 \\
$I I P 3_{D}[\mathrm{dBm}]$ & -9 & -12 & -16 \\
\hline
\end{tabular}

Table 3.

Tuning ranges for the MSA front-end.

\begin{tabular}{|ccc|}
\hline PNTR & NFTR & IIP3TR \\
\hline $21 \mathrm{~dB}$ & $12 \mathrm{~dB}$ & $7 \mathrm{~dB}$ \\
\hline
\end{tabular}

Table 4.

Required performance for the MSA-IR downconverter.

\begin{tabular}{|lccc|}
\hline Specification/Mode & Demanding & Moderate & Relaxed \\
\hline$N F_{2}[\mathrm{~dB}]$ & 12.7 & 19.75 & 28.8 \\
$I I P 3_{2}[\mathrm{dBm}]$ & 6.74 & 3.35 & -0.87 \\
\hline
\end{tabular}

achievable phase noise. Useful AFOM for mixers and amplifiers are the ranges of noise figure and the intercept point that are realized when a particular parameter, e.g., bias current, is adjusted.

Referring to Tables 1 and 2, the phase-noise tuning range (PNTR), the noise-figure tuning range (NFTR), and the 3rd-order input-intercept point tuning range (IIP3TR) are calculated in Table 3 for the complete RF front-end system.

\section{B.2. Performance Requirements for MSA-IR Down-} converter

Typical LNA $\left(N F_{1}=2 \mathrm{~dB}, I I P 3_{1}=1 \mathrm{dBm}\right.$ and gain of $\left.13 \mathrm{~dB}\right)$ and baseband circuitry ( $N F_{3}$ of $14 \mathrm{~dB}$ and $I I P 3_{3}$ of $\left.9 \mathrm{dBm}\right)$ performance parameters are assumed. With the aid of Table 2 and LNA and baseband circuits' performance assumed, the noise and linearity requirements for the MSA-IR downconverter are determined as given by Table 4 .

The NFTR and IIP3TR of the image-reject downconverter can now be determined. When the NF and IIP3 are adapted between $12.7 \mathrm{~dB}$ and $28.8 \mathrm{~dB}$, and $6.74 \mathrm{dBm}$ and $-0.87 \mathrm{dBm}$, respectively, the quadrature downconverter (e.g., with a gain of $0 \mathrm{~dB}$ ) satisfies the requirements of the considered standards with a $N F T R_{2}$ of $16 \mathrm{~dB}$ and an IIP3TR $_{2}$ of $7.6 \mathrm{~dB}$.

\section{Conclusions}

The communication devices of both today and the future will have not only to allow for a variety of applications, ranging from simple characters, via speech, audio, and graphics to video, but they will also have to maintain connection with many other devices in a variety of envi- ronments. Extension of the capabilities of wearable and wireless devices depends critically on battery endurance, as batteries continue to determine both the lifetime and size of mobile equipment.

The combination of huge functionality requirements on the one hand, and a small energy supply on the other, argues for the development of both adaptive low-power hardware and adaptive low-power software. As consumers' demands outstrip the cost benefits achieved by Moore's Law and low-power RF design, further improvements can be found in adaptivity. This eventually leads to even smaller sizes, longer standby and active times and enhanced functionality of mobile devices.

To support telecom trends such as provision of various services from different standards (text, audio, video, telephony), smooth migration towards future generation of wireless standards with higher capacities and higher data-rates for multimedia applications, and device wearability, integrated designs are required that can operate across multiple standards within one device. By sharing building blocks, adaptive multi-standard low-power RF front-ends gain advantage over their predecessors: they use a smaller chip area, consume less power, and have a potential for lower overall cost.

\section{References}

[1] A. Tasić, Adaptive RF Front-End Circuits, Ph.D. Thesis, ISBN 909019348-0, The Netherlands.

[2] ITRS roadmap, "Radio Frequency and Analog/Mixed-Signal Technologies for Wireless Communications (a section of the Process Integration Chapter) (2003)," [Online]. Available: http://www.itrs.com.

[3] GSM World, [Online]. Available: http://www.gsmworld.com/news/ press_2005/press05_21.shtml

[4] A. Abidi et al., "Power-conscious design of wireless circuits and systems," Proceedings IEEE, vol. 88, no. 10, pp. 1528-1545, Oct. 2000.

[5] M. Pedram and J. Rabaey, Power Aware Design Methodologies, Kluwer Academic Publishers, 2002.

[6] R.L. Lagendijk, Ubiquitous Communications Research Program, Final Program Report, Jan. 2002. [Online]. Available: http://www.ubicom.tudelft.nl [7] Electronics Industry Market Research and Knowledge Network, Market Research Report Number DB375. (July 2003). [Online]. Available: http://www.electronics.ca/ reports/ic/rf_ics.html\#toc

[8] T. Keller and L. Hanzo, "Adaptive multicarrier modulation: A convenient framework for time-frequency processing in wireless communications," Proceedings IEEE, vol. 88, no. 5, pp. 611-640, May 2000.

[9] W. Pasman et al., "Low-latency rendering for mobile augmented reality," Computers and Graphics, vol. 23, no. 6, pp. 875-881, 1999.

[10] A. van der Schaaf et al., "Design of an adaptive interface between video compression and transmission protocols for mobile communications," Proceedings of PV-2001, pp. 395-404, Apr. 2001.

[11] J. Pouwelse et al., "Dynamic voltage scaling on a low-power microprocessor," Proceedings Mobicom, pp. 251-259, July 2001.

[12] J. Ryynanen, K. Kivekas, J. Jussila, A. Parssinen, and K. Halonen, "A dual-band RF front-end for WCDMA and GSM applications," Proceedings CICC, pp. 175-178, May 2000.

[13] H. Hashemi et al., "Concurrent dual-band LNAs and receiver architectures," Proceedings VLSI, pp. 247-250, June 2001.

[14] A. Tasić, W.A. Serdijn and J.R. Long, "Design of multi-standard adaptive voltage controlled oscillators," IEEE Transactions on Microwave Theory and Technique, vol. 53, no. 2, pp. 556-563, Feb. 2005.

[15] X. Li and M. Ismail, "Architectures and specs help analysis of multistandard receivers," [Online]. Available: http://www.planetanalog.com/ story/OEG20030312S0038 
[16] Third Generation Partnership Project (3GPP). UE Radio Transmission and Reception (FDD) Technical Specification, 25.101, vol. 3.0.0. (Oct. 1999). [Online]. Available: http://www.3gpp.org

[17] ETSI EN 300 175-2: Digital Enhanced Cordless Telecommunications (DECT); Common Interface (CI); Part 2: Physical Layer (PHL). [Online]. Available: http://docbox.etsi.org/Reference

[18] A. Tasić, W.A. Serdijn and J.R. Long, "Adaptivity of voltagecontrolled oscillators-Theory and design," IEEE Transactions on Circuits and System-part I, vol. 52, no. 5, pp. 894-901, May 2005.

[19] D. Wang et al., "A fully integrated GSM/DCS/PCS Rx VCO with fast switching auto-band selection," Proceedings RAWCON, pp. 209-212, Aug. 2002.

[20] ETSI 300190 (GSM 05.05 version 5.4.1): Digital Cellular Communication System (Phase 2), Radio Transmission and Reception, European Telecommunications Standards Institute, Aug. 1997.

[21] IEEE standard 802.11b. Wireless Local Area Network. (1999). [Online]. Available: http://standards.ieee.org/getieee802/download/802.11b-1999_ Cor1-2001.pdf

[22] Specification of the Bluetooth System, version 1.1. (Feb. 2001). [Online]. Available: http://www.bluetooth.com

[23] A. Tasic et al., "A multi-standard adaptive image-reject downconverter," Proceedings RFIC, pp. 581-584, June 2005.

[24] M. Steyaert et al., "Low-voltage low-power CMOS-RF transceiver design," IEEE Transactions Microwave Theory and Techniques, vol. 50, no. 1, pp. 281-287, Jan. 2002.

[25] O.K. Jensen et al., "RF receiver requirements for 3G W-CDMA mobile equipment," Microwave Journal, pp. 22-46, Feb. 2000.

[26] J. Rudell et al., "An integrated GSM/DECT receiver: Design specifications," UCB Electronics Research Laboratory Memorandum, Memo no. UCB/ERL M97/82, 1998

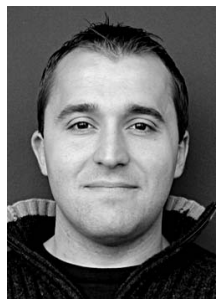

Aleksandar Tasić received the M.Sc degree in electrical engineering from the Electronics Faculty, University of Nis, Serbia, in 1998, and the Ph.D. degree from the Faculty of Electrotechnics, Mathematics and Informatics, the Delft University of Technology, the Netherlands, in 2005.

His research interest includes: design of adaptive and multi-standard RF front-end circuits for wireless communications and RF front-end system study for optimal selection of specifications for receiver building blocks.

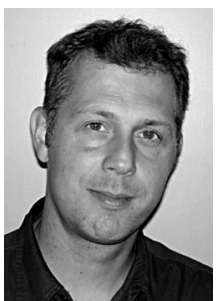

Wouter A. Serdijn was born in Zoetermeer ('Sweet Lake City'), the Netherlands, in 1966 . He started his courses at the Faculty of Electrical Engineering at the Delft University of Technology in 1984, and received the 'ingenieurs' (M.Sc.) degree in 1989. Subsequently, he joined the Electronics Research Laboratory of the same university where he received the Ph.D. in 1994.

His research interests include low-voltage, ultra-lowpower, high-frequency and dynamic-translinear analog integrated circuits along with circuits for RF and UWB wireless communications, hearing instruments and pacemakers.

Since 2002, Dr. Serdijn is a workpackage leader in the Freeband Impulse project AIR-LINK, aiming at highquality, wireless short-distance communication, employing Ultra-Wide Band radio. He is co-editor and co-author of the books Research Perspectives on
Dynamic Translinear and Log-Domain Circuits (Kluwer Academic Publishers, Boston, 2000), Low-Voltage LowPower Analog Integrated Circuits (Kluwer Academic Publishers, Boston, 1995) and Dynamic Translinear and Log-Domain Circuits (Kluwer Academic Publishers, Boston, 1998). He authored and co-authored more than 150 publications and presentations. He teaches Analog Electronics for, Micropower Analog IC Design and Electronic Design Techniques. In 2001 and 2004, he received the EE Best Teacher Award.

Dr. Serdijn has served as an Associate Editor for the IEEE Transactions on Circuits and Systems-II, as tutorial session co-chair for ISCAS'2003, as Analog Signal Processing Track Co-Chair for ISCAS'2004, as chair of the Analog Signal Processing Technical Chapter of the IEEE CAS Society, as Analog Signal Processing Track Co-Chair for ICECS'2004, as Technical Program Committee member for the 2004 International Workshop on Biomedical Circuits and Systems, and as Analog Signal Processing Track CoChair for ISCAS'2005, and currently serves as an Associate Editor for the IEEE Transactions on Circuits and SystemsI and as International Program Committee member for IASTED CDD'2005. From 2006 Wouter Serdijn will serve as a member of the Board of Governors of the Circuits and Systems Society and (again) be an Associate Editor for the IEEE Transactions on Circuits and Systems-II.

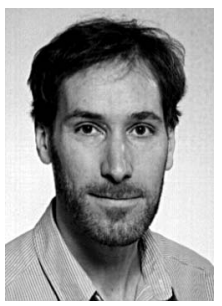

John R. Long received the B.Sc. in electrical engineering from the University of Calgary in 1984, and the M.Eng. and Ph.D. degrees in electronics engineering from Carleton University in 1992 and 1996, respectively. He was employed for 10 years by Bell-Northern Research, Ottawa (now Nortel Networks) involved in the design of ASICs for Gbit/s fiberoptic transmission systems and for 5 years at the University of Toronto. He joined the faculty at the Delft University of Technology in January 2002 as Chair of the Electronics Research Laboratory. His current research interests include: low-power transceiver circuitry for highly-integrated radio applications, and electronics design for high-speed data communications systems.

Professor Long is currently serving on the Technical Program Committees of the International Solid-State Circuits Conference (ISSCC), the European Solid-State Circuits Conference (ESSCIRC), the IEEE Bipolar/BiCMOS Circuits and Technology Meeting (BCTM), and GAAS2004 (EuMW). He is a former Associate Editor of the IEEE Journal of Solid-State Circuits. He received the NSERC Doctoral Prize and Douglas R. Colton and Governor General's Medals for research excellence, and Best Paper Awards from ISSCC 2000 and IEEE-BCTM 2003. 